\author{
Martin L. Perl, Mary A. Meyer
}

\title{
The Practice of Experimental Physics - Recollections, Reflections, and Interpretations
}

\begin{abstract}
This article is based on Martin L. Perl's Nobel Prix 1995 paper, "Reflections on the Discovery of the Tau Lepton," and is supplemented by excerpts from his presentation, "Reflections on Experimental Science," to an audience of mainly young scientists at Los Alamos National Laboratory on June 29, 1999. In both documents, Perl reflects on almost 50 years of experience in experimental science to pass on what he has learned, particularly to young people who are beginning scientific research.
\end{abstract}

\section{Introduction}

My first thoughts in writing this paper are about the young women and young men who are beginning their lives in science: students and those beginning scientific research. I have been in experimental scientific research for almost 50 years; I have done some good experiments of which the best was the discovery of the tau lepton; I have followed research directions that turned out to be uninteresting; I have worked on experiments that failed. And so, while recounting the discovery of the tau, for which I have received this great honor, the Nobel Prize, I will try to pass on what I have learned about doing experimental science.

The experimenter must take account of her or his personality and temperament in choosing problems to work on and experiments to do and apparatus to build.

Every experimenter has to understand her or his own personality and temperament as to how each of us does science. I come to science in a certain way. I was trained and worked as a chemical engineer and got into engineering 
because I love mechanical things. So my point of view in what I do, my experiments and the things I work on, are always quite mechanical, very physical. I can do simple theory but I don't think from that point of view. I am not philosophical, so I'd be the wrong person to work experiments on fundamental quantum mechanics. Each of us has to know our temperament, what our strengths are, what our interests are. And it all depends, as does everything else in life we do, on our early days.

\section{Early Days, 1930s}

About 1900 my parents came to the United States as children from what was then the Polish area of Russia. As Jews, their families left Russia to escape poverty and antiSemitism. My parents grew up in poor areas of New York City, my father in the East Side district of Manhattan and my mother in the Brownsville district of Brooklyn. My parents were determined to move into the middle class. By the time my sister Lila and I were bom in the 1920s, my father had established a printing and advertising company called Allied Printing, which brought the four of us into the middle class and kept us in the middle class through the Depression of the 1930s. We lived in the better neighborhoods of the borough of Brooklyn, and so we went to quite good schools.

\section{The experimenter must know that experimental work will sometimes require one to do work that one considers boring or demand the use of skills in which one is not particularly gifted.}

These schools and the attitude of my parents toward these schools were important in preparing me for the work of an experimental scientist. Going to school and working for good marks, indeed working for very good marks, was a serious business. I learned early to deal with an outside and sometimes hard world—good training for research work! The experimenter dealing with nature faces an outside and often hard world. Whatever the course, whether the course was boring or interesting to me, whether I was talented in mathematics or not talented in languages, my parents expected A's! This was good training for research, because large parts of experimental work are sometimes boring or involve the use of skills in which one is not particularly gifted. For example, I am not a good draftsman. Until recently when I could use computer-based drafting programs, my drawings always looked messy, with uneven lines and ragged lettering. I could never get an A in drafting in college. Yet drawing the apparatus to be built for my experiments has always been a crucial part of my experimental work.

Along with my parents' insistence, soon internalized, that I do very well in school went my love of reading and my love of mechanics. I read everything: 
fiction, history, science, mathematics, biography, travel. There were two free public libraries within walking distance of my home; I remember taking six books home from every visit, the limit set by the library. (Two books are burned in my memory, Lancelot Hogben's Mathematics for the Millions and his Science for the Citizen. I borrowed them from the library again and again and made summaries of them.) This reading had only partial approval from my parents. They wanted me to play more sports because they were acutely sensitive to their children's being one hundred percent American, and they believed that all Americans played sports and loved sports. They felt that too much reading interfered with my going outside to play sports. I loved rainy days when I did not have to go outside, and to the present I still feel very content on a rainy day.

One thing I always wanted my parents to buy for me was an Erector construction set (Figure 1), the American equivalent of Meccano or Marklin construction sets in England and Europe. But the cousin I played with every Saturday had an Erector set, and one set per extended family was considered quite enough. I loved to build with the Erector set, I loved to build toys and models out of wood, I loved to draw mechanical devices, even those I could not build. I loved to read Popular Mechanics and Popular Science. I loved all things mechanical: cars, trucks, derricks, trains, and steamships. I was in love with mechanics, and I still am. Before leaving this subject I must mention that since I never owned an Erector set as a child, I have compensated in my adult years by collecting old European, English, and American construction sets, even by devising and starting prototype production of a modem wooden construction set called BIG-NUT.

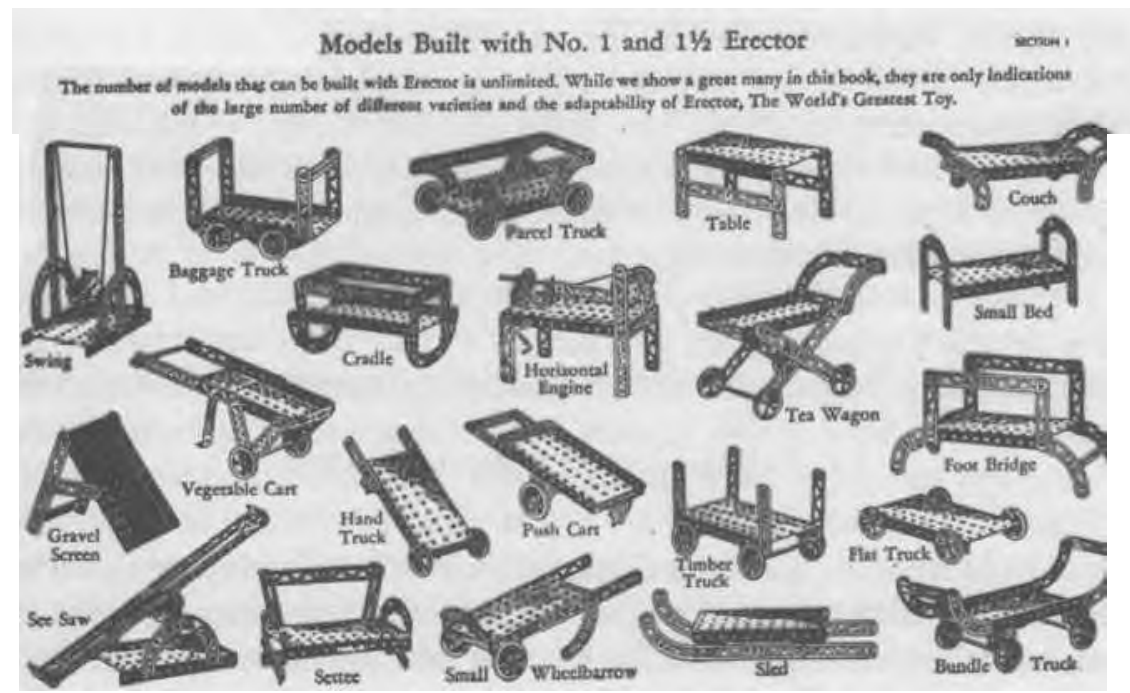

Figure 1. Models that can be built with an Erector set. 
I also was interested in chemistry, but my parents were not willing to buy me a chemistry set. I had some chemicals, but when I bought sulfuric acid and nitric acid, my father confiscated the acids on the grounds of safety. As every child knows, chemistry with nothing stronger than vinegar soon becomes dull. Strangely, for a person who became a physicist, I was not interested in amateur radio or in building radios. I don't know why. This was the 1930s when vacuum tubes and variable condensers made radio building quite mechanical.

In spite of very good school marks, a love of books (particularly in science and mathematics), and a great love of mechanics, I never thought of becoming a scientist. That was because, as the children of immigrants, my sister and I were taught that we must use our education to "earn a good living." In fact, we didn't have to be taught that. It was obvious to us. Although I won the physics medal when I graduated from high school, I did not think of becoming a physicist or any kind of scientist. My parents and I knew about a few scientists, certainly Pasteur, and perhaps Einstein, but we did not know that it was possible for someone to earn a living as a scientist.

We did know that a person could earn a living as an engineer. And so in choosing a profession for me, my parents and I took into account my love of mechanics and science and mathematics, and we decided on engineering, an unusual choice for a Jewish boy in the early 1940s because there was still plenty of anti-Semitism in engineering companies. I enrolled in the Polytechnic Institute of Brooklyn, now Polytechnic University, and began studying chemical engineering. There were several reasons for choosing chemical engineering. Chemistry was a very exciting field in the late 1930s and early 1940s. There would always be a good job in chemical engineering.

My studies were interrupted by the war. When the war ended, I returned to the Polytechnic Institute and received a summa cum laude bachelor's degree in chemical engineering in 1948. The skills and knowledge I acquired at the Polytechnic Institute have been crucial in all my experimental work, including the use of strength-of-materials principles in equipment design, machine shop practice, and engineering drawing.

\section{Learning the Engineer's and Physicist's Trades, 1940s and 1950s}

\subsection{Engineering at General Electric}

Upon graduation, I joined the General Electric Company. After a year in an advanced engineering training program, I settled in Schenectady, New York, working as a chemical engineer in the electron tube production factory. Our job was to troubleshoot production problems, to improve production processes, and occasionally to do a little development work. We were not a fancy $R \& D$ office. 


\section{It is often impossible to predict the future of a technology used in engineering or science.}

At General Electric, I learned my first lesson about technology and trying to predict the future. We made receiver tubes, and I had a boss who had the idea that we should try to make very small vacuum tubes for portable radios. The idea was that the radio would turn on immediately; one would not have to wait for the filament to warm up. People my age know about this delay. My boss could see, for marketing purposes, the value of having radios turn on immediately. This was 1948 to 1950, and not so far away, at Bell Laboratories in New Jersey, the transistor was invented. At the GE research laboratories, however, we had not foreseen that the vacuum tube was the wrong direction, that the transistor was the right direction.

This is what you have to know in developing technology - that it's impossible to predict the future of a technology. You have to keep your eyes open. You can never say what is going to come in the future, and that's important in research or whatever you do.

For my job I had to learn a little about how electron vacuum tubes worked, so I took a few courses at Union College in Schenectady, specifically, atomic physics and advanced calculus. I got to know a wonderful physics professor, Vladimir Rojansky. One day he said to me "Martin, what you are interested in is called physics, not chemistry!" At the age of 23, I finally decided to begin the study of physics.

\subsection{Atomic Physics at Columbia under 1.1. Rabi}

Just as the Polytechnic Institute was crucial in my learning how to do engineering, just as Union College and Vladimir Rojansky were crucial in my choosing physics, so Columbia University and my thesis advisor, I. I. Rabi, were crucial in my learning how to do experimental physics. I entered the physics doctoral program at Columbia University in the autumn of 1950. Looking back, it seems amazing that I was admitted. True, I had a summa cum laude bachelor's degree, but I had taken only two courses in physics: one year of elementary physics and a half-year of atomic physics. There were several reasons why I could do this in 1950; it could not have been done today. First, graduate study in physics was primitive in 1950, compared to today's standards. We did not study quantum mechanics until the second year; the first year was devoted completely to classical physics. The most advanced quantum mechanics we ever studied was a little bit in Heitler, and we were not expected to be able to do calculations in quantum electrodynamics.

Second, there was no thought of advising or course guidance by the Columbia Physics Department faculty—students were on their own. I was arrogant about 
my ability to leam anything fast. By the time I realized I was in trouble, that many of my fellow students were both smarter and better trained than I, it was too late to quit. I survived the Columbia Physics Department, never the best student, but an ambitious and hardworking student. I was married and had a child. I had to get my Ph.D. and once more earn a living.

I undertook for my doctoral research the problem of using the atomic-beam resonance method to measure the quadrupole moment of the sodium nucleus. (The atomic-beam resonance method was invented by Rabi, for which he had received a Nobel Prize in 1944.) This quadrupole measurement had to be made using an excited atomic state and Rabi had found a way to do this. My experimental apparatus (Figure 2) was boldly mechanical, with a brass vacuum chamber, a physical beam of sodium atoms, submarine storage batteries to power the magnets and, in the beginning of the experiment, a wall galvanometer to measure the beam current. I developed much of my style in experimental science in the course of this thesis experiment. When designing the experiment and when thinking about the physics, the mechanical view is always dominant in my mind. More importantly, my thinking about elementary particles is physical and mechanical. In the basic production process for tau leptons,

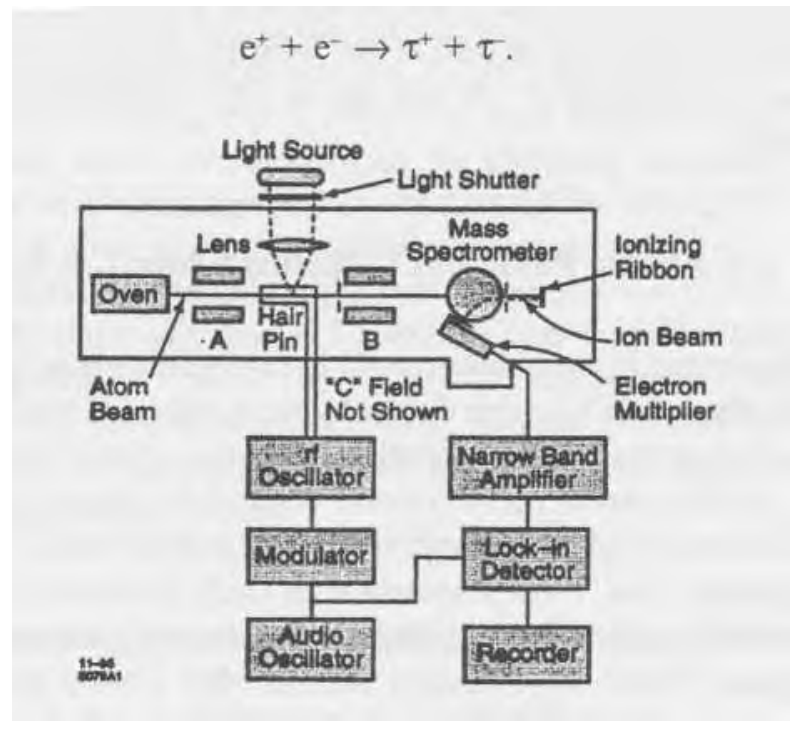

Figure 2. From the author's Ph.D. thesis experiment in atomic beams (Perl, Rabi, and Senitzky 1955). The caption read, "Schematic drawing of the apparatus. The light source is shown on the side of the apparatus for clarity, but it actually lies above the apparatus. The $\mathrm{C}$ magnet, which produces a homogeneous field in the "hairpin," is not shown for clarity. The six external boxes which represent the major electronic components do not indicate the physical position of the components." 
I see the positron, $\mathrm{e}^{+}$, and electron, $\mathrm{e}^{\sim}$, as tiny particles that collide and annihilate one another. I see a tiny cloud of energy formed which we technically call a virtual photon, $\mathrm{Y}_{\mathrm{vjrtual}}$; and then I see that energy cloud change into two tiny particles of new matter-a positive tau lepton, $\mathrm{T}^{+}$, and a negative tau lepton, $T$.

\section{Be aware of the emotional roller coaster of research.}

In my thesis experiment I first experienced the pleasures, the anxieties and, sometimes, the pain that are inherent in experimental work: the pleasure when an experiment is completed and data safely recorded, the anxiety when an experiment does not work well or something breaks, the pain when an experiment fails or when an experimenter does something stupid. In my thesis experiment the acquisition of a set of data took about a day, and so there were several alternating periods of anxiety and pleasure within a week. When I broke a McCloud vacuum gauge and spread mercury inside the vacuum chamber, the pain of restoring the apparatus lasted but a few weeks. At the other extreme, in the discovery of the tau, the ups and downs of my emotions extended over years. As in the course work, I was on my own in learning the experimenter's trade. I learned quickly — as I tell my graduate students now, there are no answers in the back of the book when the equipment doesn't work or the measurements look strange.

\section{It is best to use your own ideas for and in experiments.}

I learned things more precious than experimental techniques from Rabi. I learned the deep importance of choosing one's own research problems. Rabi once told me that he would worry when talking to Leo Szilard that Szilard would propose some idea to Rabi. This was because Rabi wouldn't carry out an idea suggested by someone else, even though he had already been thinking about that same idea.

I also learned from Rabi the importance of getting the right answer and checking it thoroughly. It was Rabi who always emphasized the importance of working on a fundamental problem, and it was Rabi who sent me into elementary particle physics. It would have been natural for me to continue in atomic physics, but he preached particle physics to me-particularly when his colleagues in atomic physics were in the room. I think that most of that public preaching may have been Rabi's way of deliberately irritating his colleagues.

\section{Avoid fast talkers and fast thinkers.}

The most important thing I learned from Rabi is to avoid fast talkers and fast thinkers. This is very important for young people. I try to avoid working with fast talkers and thinkers. I don't mind working with fast thinkers if they say very little. And the best thing of all is to work with people who are smarter than you, who think faster than you, but never say anything. 


\section{Choose the group or department where you will have the most freedom.}

I followed a two-part theorem that I always pass on to my graduate students and postdoctoral research associates: (1) Don't choose the most powerful experimental group or department - choose the group or department where you will have the most freedom; and (2) There is an advantage in working in a small or new group: you will get the credit for what you accomplish. When I received my Ph.D. in 1955, I had job offers from the physics departments at Yale, the University of Illinois, and the University of Michigan. At that time, Yale and Illinois had better reputations in elementary particle physics, so I deliberately went to Michigan.

\subsection{High-Energy Physics at Michigan}

\section{You don't have to know everything-you can learn a subject or a technology when you need it.}

At Michigan, I made a change from atomic physics to high-energy physics, and I learned something else that I've always stuck to, though it's sometimes gotten me into trouble. You don't have to know everything. Science is normally very specialized, but don't make the mistake of studying for the new subject in great detail. You'll have time later to catch up and learn; you can take courses and talk to your colleagues (as long as they're not fast talkers).

At Michigan I first worked in bubble chamber physics with Donald Glassier. But I wanted to be on my own. When the Russians flew SPUTNIK in 1957, I saw the opportunity and jointly with my colleague, Lawrence W. Jones, wrote to Washington for research money. We began our own research program, using first the now-forgotten luminescent chamber and then the spark chambers. Jones and I, using spark chambers, carried out at the Bevatron a neat set of measurements on the elastic scattering of pions on protons (Damouth, Jones, and Perl 1963; Perl, Jones, and Ting 1963).

In eight wonderful and productive years at the University of Michigan, I learned the experimental techniques of research in elementary particle physics (scintillation counters, bubble chamber, trigger electronics, and data analysis) working with my research companions Lawrence Jones, Donald Meyer, and later, Michael Longo. We learned these techniques together, often adding our own new developments. One of the most pleasurable experiences was the development of the luminescent chamber by Jones and me with the help of our student Kwan Lai (Lai, Jones, and Perl 1961). We photographed and recorded the tracks of charged particles in a sodium iodide crystal using primitive electron tubes that intensified the light coming from the track. 


\section{The Electron-Muon Problem, 1960s}

Later, after I left Michigan for Stanford University, Longo and I, working with my student Michael Kreisler, initiated a novel way to measure the elastic scattering of neutrons on protons (Kreisler et al. 1966). These elastic scattering experiments pleased me in many ways. The equipment was bold and mechanical, with large flashing spark chambers and a camera with a special mechanism for quick movement of the film. Data acquisition was fast, and the final data were easily summarized in a few graphs.

I gradually became dissatisfied with the theory needed to explain our measurement. I am a competent mathematician, but I dislike complex mathematical explanations and theories, and in the 1950s and 1960s the theory of strong interactions was a complex mess, going nowhere. I began to think about the electron and the muon, elementary particles that do not participate in the strong interaction.

\subsection{Properties of the Electron and Muon}

The electron was discovered in the late nineteenth century; the final characterization of its nature was achieved by J. J. Thomson in the 1890s. He received a Nobel Prize in 1906 for his investigation of electrical conduction in gases. The muon was found in cosmic rays in the 1930s. Table 1 lists the properties of electrons and muons as they were known in the 1960s; this table is still correct today. (Table 2 lists what was known about leptons and quarks in the 1960s.)

Table 1. Properties of the electron and the muon as know in the 1960s. The electric charge is given in units of $1.60 \times 10^{19}$ coulombs. The mass is given in units of the mass of the electron $9.11 \times 10^{\prime 31}$ kilograms.

\begin{tabular}{|l|c|c|}
\hline Particle & Electron & Muon \\
\hline Symbol & $\mathrm{e}$ & $\mu$ \\
\hline Electric charge & +1 or -1 & +1 or -1 \\
\hline Mass & 1 & 206.8 \\
\hline Does particle have electromagnetic interactions? & yes & yes \\
\hline Does particle have weak interactions? & yes & yes \\
\hline
\end{tabular}


Table 1, cont.

\begin{tabular}{|l|c|c|}
\hline Particle & Electron & Muon \\
\hline Does particle have strong interactions? & no & no \\
\hline Associated neutrino & $v_{e}$ & $v_{\mu}$ \\
\hline Associated antineutrino & $v_{e}$ & $v_{\mu}$ \\
\hline Lifetime & Stable & $2.2 \times 10^{-6} \mathrm{sec}$ \\
\hline
\end{tabular}

Table 2. What We Knew About Leptons and Quarks in the 1960s

\begin{tabular}{|l|c|c|}
\hline \multicolumn{2}{|c|}{ Leptons } \\
\hline Particle & Electric Charge & Mass \\
\hline Electron (e) & -1 & About 0.5 \\
\hline Electron neutrino $\left(\mathrm{v}_{\mathrm{e}}\right)$ & 0 & Close to 0 or 0 \\
\hline Muon (p) & -1 & About 105 MeV \\
\hline Muon neutrino $\left(\mathrm{v}_{\mathrm{p}}\right)$ & & Close to 0 or 0 \\
\hline & Quarks & Mass \\
\hline Particle & Electric Charge & About 2 MeV \\
\hline Up (u) & $+2 / 3$ & About 4 MeV \\
\hline Down (d) & $-1 / 3$ & About $105 \mathrm{MeV}$ \\
\hline Strange (s) & $-1 / 3$ &
\end{tabular}

There were two puzzles about the relation between the electron and the muon. First, as shown in Table 1, the properties with respect to particle interactions are the same for the electron and the muon, but the muon is 206.8 times heavier. Why? The second puzzle is that since the muon is unstable, with an average lifetime of $2.2^{\prime} 10^{\prime 6}$ seconds decay to an electron, one expects that the decay process would be

$$
\begin{aligned}
& \mu^{-} \rightarrow \mathrm{e}^{-}+\gamma \\
& \mu^{+} \rightarrow \mathrm{e}^{+}+\gamma .
\end{aligned}
$$

Here, y means a photon, and the expectation would be that the y carries off the excess energy produced by the difference between the muon mass and the 
electron mass - but this is not the nature of the muon or the electron. The muon decays to an electron by a complicated process,

$$
\begin{aligned}
& \mu^{-} \rightarrow \mathrm{e}^{-}+\bar{v}_{\mathrm{e}}+\mathrm{n}_{\mu} \\
& \mu^{+} \rightarrow \mathrm{e}^{+}+\mathrm{n}_{\mathrm{e}}+\bar{\nu}_{\mu},
\end{aligned}
$$

in which a neutrino and an antineutrino are produced. There is something in the nature of the muon that is different from the nature of the electron.

While at the University of Michigan, I was intrigued by the careful measurements being made on the (g-2) of the muon by Charpak et al. (1962) at CERN and on the (g-2) of the electron by Wilkinson and Crane (1963) at Michigan. The quantity (g-2) measures the magnetic properties of a particle. I was also interested in the precision studies of positronium and muonium then in progress, as well as other precision atomic physics experiments. These low- energy studies of the charged leptons were in very capable hands, and I could not see how I could contribute. I knew about the pioneer low-energy neutrino experiments of Frederick Reines and Clyde Cowan, Jr., who shared the Nobel Prize with me in 1995. As for high-energy neutrino experiments, they were already being carried out by the powerful set of Nobel Laureates Leon Lederman, Melvin Schwartz, and Jack Steinberger (Danby et al. 1962).

\section{Stay away from lines of research where very smart and competent people are working.}

I reflected that it would be most useful for me to consider high-energy experiments on charged leptons, experiments that might clarify the nature of the lepton or explain the electron-muon problem. This is a research strategy that I have followed quite a few times in my life. I stay away from lines of research where many people are working, and, in particular, I stay away from lines of research where very smart and competent people are working. I find it more comfortable to work in uncrowded areas of physics. I caution the young scientist with this advice. Almost all the time, the best experimenters and the majority of the experimenters work in the most fruitful areas. (If there are few or no investigators working on a problem, it may be an unproductive problem.) In the end, it is a question of temperament, comfort, and judgment.

\section{The Discovery of the Tau Lepton, 1960s and 1970s}

\subsection{SLAC, Leptons, and Heavy Leptons}

The opportunity arose to think seriously about high-energy experiments on charged leptons in 1962 when Wolfgang K. H. Panofsky and Joseph Ballam offered 
me a position at the yet-to-be-built Stanford Linear Accelerator Center (SLAC). Here was a laboratory that would have primary electron beams: a laboratory at which one could easily obtain a good muon beam and a laboratory at which one could easily obtain a good photon beam for producing lepton pairs. Furthermore, on the Stanford campus at the High-Energy Physics Laboratory, the Princeton- Stanford $\mathrm{e}^{-} \mathrm{e}^{-}$storage ring was operating (O'Neil et al. 1958; Barber et al. 1966).

When I arrived at SLAC in 1963, I began to plan various attacks on, and investigations of, the electron-muon problem. Although the linear accelerator would not begin operation until 1966, my colleagues and I began to design and build experimental equipment. The proposed attacks and investigations were of two classes. In one class, I proposed to look for unknown differences between the electron and the muon, the only known differences being the mass difference

and the observation that the decay reaction $\mu \rightarrow \mathrm{e}+\gamma$ does not occur. The othes class of proposed attacks and investigations was based on my speculation that there might be more leptons similar to the electron and the muon, unknown heavier charged leptons. I dreamed that if we could find a new lepton, the properties of the new lepton might teach us the secret of the electron-muon puzzle.

My first attack used an obvious idea (Figure 3). An intense photon (y) beam could be made at SLAC using the reactions

$$
\mathrm{e}^{-}+\text {nucleus } \rightarrow \gamma+\ldots
$$

The photons so produced could then interact with another nucleus to produce a pair of charged particles: $\mathrm{x}^{+}$and $\mathrm{x}^{-}$,

$$
\gamma+\text { nucleus } \rightarrow x^{+}+x^{-}+\ldots
$$

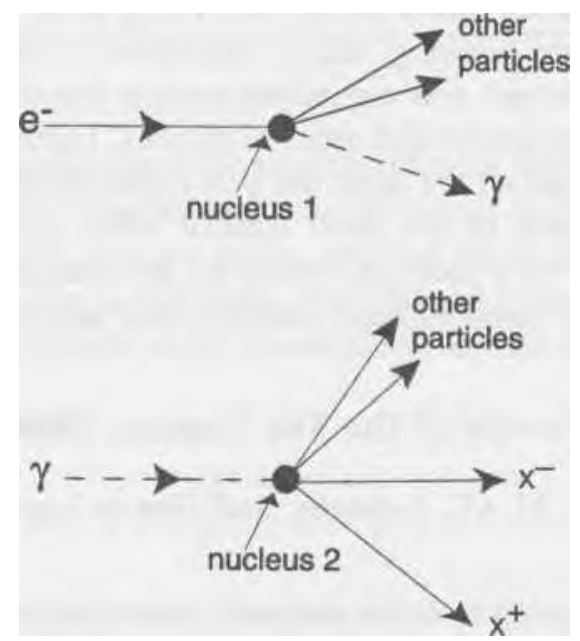

Figure 3. Graphic representation of Eq. 5. 
Any pair of charged particles could be produced if the y had enough energy. To the young experimenter, I remark that there is nothing wrong with an obvious experimental idea as long as you are the first to use the idea. My hope was that we would find a new $\mathrm{x}$ particle, perhaps a new charged lepton somehow related to the electron or muon, a vague hope by the standards of our knowledge of elementary particle physics today. We were certainly naive in the 1960s. We didn't find any new leptons or any new particles of any kind (Bama et al. 1968); as we now know, there were no new particles to find given the experimental limitations of this search experiment. The search used the pair-production calculations of Tsai and Whitis (1966); this experiment was the beginning of a long and fruitful collaboration between my colleague Y.-S. (Paul) Tsai and myself.

\subsection{Studies of Muon-Proton Inelastic Scattering}

Although this first attempt to penetrate the mysteries of the electron and muon failed, we were already preparing to study muon-proton inelastic scattering, $\mu^{-}+p \rightarrow \mu^{-}+$hadrons (elementary particles that have strong interactions), to compare it with electron-proton inelastic scattering, $\mathrm{e}^{-}+\mathrm{p} \longrightarrow \mathrm{e}^{-}+$hadrons. Extensive studies of e-p inelastic scattering were planned at SLAC. Indeed, some of those studies led to the awarding of the 1990 Nobel Physics Prize to Jerome Friedman, Henry Kendall, and Richard Taylor. My hope was that we would find a difference between the $\mathrm{p}$ and e other than the differences of mass and lepton number. In particular, I hoped that we would find a difference at large momentum transfers-another naive hope when viewed by our knowledge today of particle physics. For example, I speculated (Perl 1971) that the muon might have a special interaction with hadrons not possessed by the electron (see Figure 4).

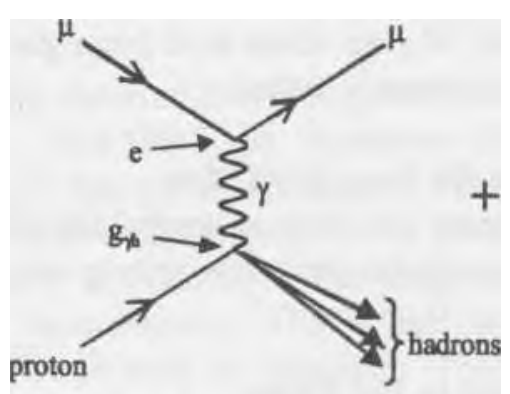

One-photon exchange

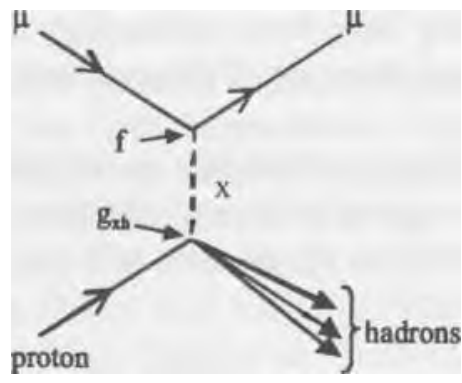

One-X exchange

Figure 4. The interaction of a muon with hadrons through exchange of a particle $\mathrm{x}$, an example of the speculation that the muon has a special interaction with hadrons that is not possessed by the electron (Perl 1971). 
Returning to the late 1960s, my colleagues and I measured the differential cross sections for inelastic scattering of muons on protons and then compared the p-p cross sections with the corresponding e-p cross sections (Toner et al. 1972; Braunstein et al. 1972). We were looking for a difference in magnitude or a difference in behavior of the cross sections. As discussed in Perl and Rapidis (1974), these differences could come from a new nonelectromagnetic interaction between the $\mathrm{p}$ and hadrons or from the $\mathrm{p}$ not being a point particle. However, as summarized in Toner et al. (1972), we found no significant deviation.

Other experimenters studied the differential cross section for $\mathrm{p}-\mathrm{p}$ elastic scattering and compared it with e-p elastic scattering (Ellsworth et al. 1960; Camilleri et al. 1969: Kostoulas et al. 1974). but statistically significant differences between $\mu-p$ and $e-p$ cross sections could not be found in either the elastic or inelastic case. Furthermore, there were systematic errors on the order of $5 \%$ or $10 \%$ in comparing $\mathrm{p}-\mathrm{p}$ and e-p cross sections because the techniques used were so different.

\section{Consider the art of obsession in science and engineering.}

Experimental science is a craft and an art, and part of the art is knowing when to end a fruitless experiment. There is a danger of becoming obsessed with an experiment even if it goes nowhere. At some point, you've got to say "I really don't know how to improve this." I avoided obsession and gave up on the scattering experiment. That turned out to be a good decision because modem experiments have shown that the scattering experiment does not illuminate any differences between the electron and the muon beyond the mass difference. Bright young people are always following you into the field. They'll be as smart as you because human intelligence has not changed in tens of thousands of years; they'll be better trained than you because training is improving all the time; and they'll certainly have better technology than you. If your ideas have been good, they will pick them up. If they weren't good, it doesn't matter.

\section{Expect to have five or ten bad ideas for every good idea.}

It's going to happen like that. The reason you have to control the obsession is that if you obsess, you will not get through the ideas that are not so good.

\subsection{Heavy Leptons in the $1960 \mathrm{~s}$}

While building the apparatus using our muon-proton inelastic scattering experiment, and during the first operation of that experiment, I was thinking of another way to look for new charged leptons, $\mathrm{L}$, using the reaction 


$$
\mathrm{e}^{+}+\mathrm{e}^{-} \rightarrow \mathrm{L}^{+}+\mathrm{L}^{-} \text {. }
$$

Before turning to a third attack on the electron-muon problem, I will describe the general thinking in the 1960s about the possible existence of and types of new leptons. By the beginning of the 1960s, papers had been written on the possibility of the existence of charged leptons more massive than the e and p. I remember reading the 1963-1964 papers of Zel'dovich (1963), Lipmanov (1964), and Okun (1965). Since the particle generation concept was not yet an axiom of our field, older models of particle relationships were used. For example, if one thought (Low 1965) that there might be an electromagnetic excited state $\mathrm{e}^{*}$ of the $\mathrm{e}$, then the proper search method was

$$
\begin{gathered}
\mathrm{e}^{-}+\text {nucleon } \rightarrow \mathrm{e}^{-*}+\ldots, \\
\mathrm{e}^{-*} \rightarrow \mathrm{e}^{-}+\gamma .
\end{gathered}
$$

If one thought (Lipmanov 1964) that there was a $\mu$ that was a member of a $\mu, v_{\mu}, \mu$ ' family, then the proper search method was

$$
v_{\mu}+\text { nucleon } \rightarrow \mu,+\ldots \text {. }
$$

It is interesting to note, in view of the search a decade later for $\tau \rightarrow \nu_{t} \pi$, that Lipmanov (1964) calculated the branching fraction for this decay mode. By the second half of the 1960s, the concept had been developed of a heavy lepton $L$ and its neutrino $\mathbf{v}_{1}$ forming an $\mathrm{L}, \mathrm{V}_{\mathrm{L}}$ pair. Thus, in a paper written in 1968, Rothe and Wolsky (1969) discuss the lower mass limit on such a lepton set by its absence in $K$ decays. They also discuss the decay of such a lepton into the modes

$$
\mathrm{L} \rightarrow \mathrm{e} \bar{v}_{\mathrm{e}} \nu_{\mathrm{L}}, \mu \bar{v}_{\mu} v_{\mathrm{L}}, \pi v_{\mathrm{L}} .
$$

The construction and operation of electron-positron colliders began in the 1960s (Voss 1994). By September 1967 at the Sixth International Conference on High Energy Accelerators, Howard (1967) was able to list quite a few electron-positron colliders. The pioneer 500-MeV ADA collider was already operating at Frascati in the early 1960s, and, also at Frascati, ADONE was under construction. The 1-GeV ACO at Orsay and 1.4-GeV VEPP-2 at Novosibirsk were in operation. The 6-GeV CEA Collider at Cambridge was being tested, and colliders had been proposed at DESY and SLAC (Ritson et al. 1964). The 1964 SLAC proposal (Ritson et al. 1964) (see Figure 5) had already discussed the reaction

$$
\mathrm{e}^{+}+\mathrm{e}^{-} \rightarrow \mathrm{x}^{+}+\mathrm{x}
$$


Of course, $\mathrm{x}$ might be a charged lepton. This proposal did not lead directly to the construction of an $\mathrm{e}^{+} \mathrm{e}^{-}$collider at SLAC because we could not get the funding. About five years later-with the steadfast support of the SLAC director, Wolfgang Panofsky, and with a design and construction team led by Burton Richter-construction of the SPEAR $\mathrm{e}^{+} \mathrm{e}^{-}$collider was begun at SLAC.

It was this 1964 proposal and the 1961 seminal paper of Cabibbo and Gatto (1961) that focused my thinking on new charged lepton searches using an $\mathrm{e}^{+} \mathrm{e}^{-}$collider. As we carried out the experiments described previously, I kept looking for a model for new leptons - a model that would lead to definitive colliding beam searches, while remaining reasonably general. Helped by discussions with colleagues such as Paul Tsai and Gary Feldman, I came to what I later called the sequential lepton model. I thought of a sequence of pairs

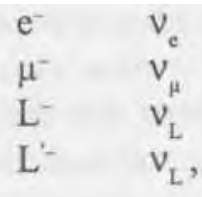

each pair having a unique lepton number. I usually thought about the leptons as being point Dirac particles. Of course, the assumptions of unique lepton number and point particle nature were not crucial, but I liked the simplicity. After all, I had turned to lepton physics in the early 1960 s in a search for simple physics. The idea was to look for

$$
\mathrm{e}^{+}+\mathrm{e}^{-} \rightarrow \mathrm{L}^{+}+\mathrm{L}^{-}
$$

with

$$
\begin{aligned}
& \mathrm{L}^{+} \rightarrow \mathrm{e}^{+}+\text {undetected neutrinos carrying off energy } \\
& \mathrm{L}^{-} \rightarrow \mu^{-}+\text {undetected neutrinos carrying off energy, } \\
& \mathrm{L}^{+} \rightarrow \mu^{+}+\text {undetected neutrinos carrying off energy } \\
& \mathrm{L}^{-} \rightarrow \mathrm{e}^{-}+\text {undetected neutrinos carrying off energy. }
\end{aligned}
$$

This search method had many attractive features:

- If the L was a point particle, we could search up to an L mass almost equal to the beam energy, if we had enough luminosity.

- The appearance of an $\mathrm{e}^{+} \mu^{-}$or $\mathrm{e}^{-} \mu^{+}$event with missing energy would be dramatic.

- The apparatus we proposed to use to detect the reactions in Eqs. 10 would be very poor in identifying types of charged particles (certainly by today's standards), but the easiest particles to identify were the e and the $\mu$. 
- There was little theory involved in predicting that the $\mathrm{L}$ would have the weak decays

$$
\begin{aligned}
& \mathrm{L}^{-} \rightarrow v_{\mathrm{L}}+\mathrm{e}^{-}+v_{\mathrm{e}} \\
& \mathrm{L}^{-} \rightarrow v_{\mathrm{L}^{+}}+\mu^{-}+v_{\mu}
\end{aligned}
$$

with corresponding decays for the L-. One simply could argue by analogy from the known decay that

$$
\mu^{-} \rightarrow v_{\mu}+\mathrm{e}^{-}+v_{\mathrm{e}}
$$

\title{
I incorporated the search method summarized by Eqs. 10 in our 1971 SLAC-
} LBL proposal to use the not-yet-completed SPEAR $\mathrm{e}^{+} \mathrm{e}^{-}$storage ring.

My thinking about sequential leptons and the use of the method of Eqs. 10 to search for them was greatly helped and influenced by two seminal papers of Paul Tsai. In 1965, he published with Anthony Hearn the paper, "Differential Cross Section for $\mathrm{e}^{+}+\mathrm{e}^{-} \rightarrow \mathrm{W}^{+}+\mathrm{W}^{-} \rightarrow \mathrm{e}^{-}+\bar{\nu}_{\mathrm{e}}+\mu^{+}+\mathrm{v}_{\mathrm{u}}$ " (Tsai and Hearn 1965). This work discussed finding vector boson pairs $\mathrm{W}^{+} \mathrm{W}$ by their eg decay mode. It was thus closely related to my thinking, described above, of finding $L^{+} L^{\prime}$ pairs by their eg decay mode. Tsai's 1971 paper provided the detailed theory for applying the sequential lepton model to our actual searches (Tsai 1971). Thacker and Sakurai (1971) also published a paper on the theory of sequential lepton decays, but it is not as comprehensive as the work of Tsai. Also important to me was the general paper by James Bjorken and Chris Llewellyn-Smith (1973), "Spontaneously Broken Gauge Theories of Weak Interactions and Heavy Leptons."

\author{
PROPOSAL FOR A HIGH-ENERGY \\ ELECTRON-POSITRON COLLIDING-BEAM STORAGE RING \\ AT THE \\ STANFORD LINEAR ACCELERATOR
}

March 1964

\author{
It is proposed that the Atomic Energy Commission support the construction at Stanford \\ University of a Colliding-Beam Facility (storage ring) for high-energy electrons and \\ positrons. This facility would be located at the Stanford Linear Accelerator Center, and it \\ would make use of the SLAC accelerator as an injector. \\ This proposal was prepared by the following persons: \\ Stanford Physics Department \\ D. Ritson \\ Stanford Linear Accelerator Center \\ S. Berman, A. Boyarski, F. Bulos, E. L. Garwin, \\ W. Kirk, B. Richter, and M. Sands
}

Figure 5. The cover page of the 1964 SLAC proposal to build an electron-positron collider (Ritson et al.

1964). 


\subsection{Searching for Leptons}

After numerous funding delays, a group led by Burton Richter and John Rees of SLAC Group C began to build the SPEAR e'e collider at the end of the 1960s. Gary Feldman and I, along with our Group E, joined with their Group C and a Lawrence Berkeley Laboratory group led by William Chinowsky, Gerson Goldhaber, and George Trilling to build the SLAC-LBL detector. In 1971, we submitted the SLAC-LBL Proposal (Larsen et al. 1971) using the SLAC-LBL detector at SPEAR. The contents of the proposal consisted of five sections (Introduction, Boson Form Factors, Baryon Form Factors, Inelastic Reactions, Search for Heavy Leptons), followed by figure captions, references, and the Supplement.

The heavy lepton search was left for last and allotted just three pages because to most others it seemed a remote dream. But the three pages did contain the essential idea of searching for heavy leptons using e-p events, Eqs. 10.

I wanted to include a lot more about heavy leptons and the e-p problem, but my colleagues thought that would unbalance the proposal. We compromised on a 10-page supplement titled, "Supplement to Proposal SP-2 on Searches for Heavy Leptons and Anomalous Lepton-Hadron Interactions," which began as follows:

While the detector is being used to study hadronic production processes it is possible to simultaneously collect data relevant to the following questions:

- Are there charged leptons with masses greater than that of the muon?

- Are there anomalous interactions between the charged leptons and the hadrons?

My first interest was to look for heavy leptons, but I still had my old interest of looking for an anomalous lepton interaction, the idea that led to the study of muon-proton inelastic scattering. While SPEAR and the SLAC-LBL detector were being built, lepton searches were being carried out at the ADONE $\mathrm{e}^{+} \mathrm{e}^{-}$storage ring by two groups of experimenters in electron-positron annihilation physics. One group reported in 1970 and 1973 (AllesBorelli et al. 1970; Bemardini et al. 1973). In the later paper, they searched up to a mass of about $1 \mathrm{GeV}$ for a conventional heavy lepton and up to about $1.4 \mathrm{GeV}$ for a heavy lepton with decays restricted to leptonie modes. The other group of experimenters in electronpositron annihilation physics was led by Shuji Orito and Marcello Conversi. Their search region (Orito et al. 1974) also extended to masses of about $1 \mathrm{GeV}$.

The SPEAR $\mathrm{e}^{+} \mathrm{e}^{-}$collider began operation in 1973. Eventually SPEAR obtained a total energy of about $8 \mathrm{GeV}$; but in the first few years, the maximum energy with useful luminosity was $4.8 \mathrm{GeV}$. We began operating the SLAC-LBL 
experiment in 1973 in the form shown in Figure 6. The SLAC-LBL detector was one of the first large-solid-angle, general-purpose detectors built for colliding beams. The use of largesolid-angle particle tracking and the use of large-solid- angle particle identification systems are obvious now, but it was not obvious twenty years ago. The electron detection system used lead-scintillator sandwich counters built by our Berkeley colleagues. The muon detection system was also crude, using the iron flux return, which was only 1.7 absorption lengths thick.

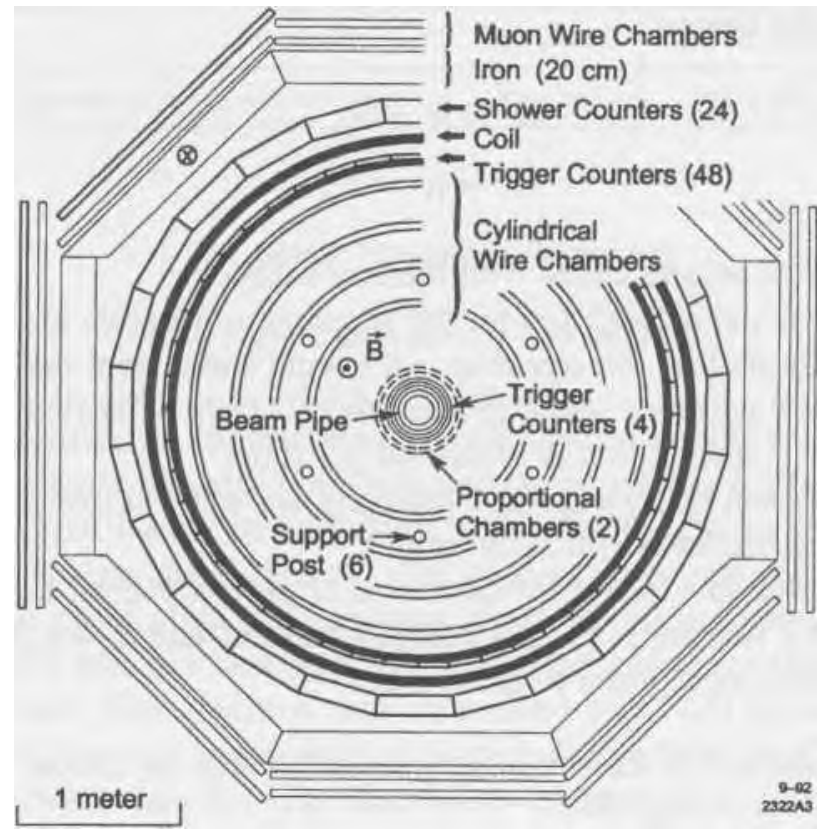

In June 1975, I gave the first international talk on the e- $\mu$ events at the 197: Summer School of the Canadian Institute for Particle Physics (Perl 1975a). This was the second of my two lectures on electron-positron annihilation at the school, and it had two purposes: (1) to discuss possible sources of e-p events: heavy leptons, heavy mesons, or intermediate bosons, and (2) to demonstrate that we

had good evidence for e- $\mu$ events. The largest single energy data sample (Table 3) was at $4.8 \mathrm{GeV}$, the highest energy at which we could then run SPEAR. The $24 \mathrm{e}-\mathrm{p}$ events in the "total charge $=0$; number photons $=0$ " column was the strongest evidence at that time for the $\mathrm{T}$. One of the cornerstones of this claim was an

informal analysis carried out by Jasper Kirkby, who was then at Stanford University and at SLAC. He showed me that by just using the numbers in the 0 - charge/O-photons columns of Table 3, we could calculate the probabilities for 
hadron misidentification in this class of events. There were not enough $\mathrm{e}-\mathrm{h}, \mathrm{p}-\mathrm{h}$, and $\mathrm{h}-\mathrm{h}$ events to explain away the 24 e-p events. The misidentification probabilities determined from three-or-more-prong hadronic events and other considerations are given in Table 4. Compared to present experimental techniques,

the $\mathrm{P}_{\mathrm{h} \rightarrow \mathrm{e}}$ and $\mathrm{P}_{\mathrm{h} \rightarrow \mathrm{u}}$ misidentification probabilities of about 0.2 are enormous, but I could still show that the 24 e-p events could not be explained away. And so the evidence for a new phenomenon was quite strong - not incontrovertible, but still strong. The new phenomenon was a sequential heavy lepton — a new heavy meson-with the decays

$$
\begin{aligned}
& \mathrm{M}^{-} \rightarrow \mathrm{e}^{-}+\bar{\nu}_{\mathrm{e}} \\
& \mathrm{M}^{-} \rightarrow \mu^{-}+\bar{\nu}_{\mu} .
\end{aligned}
$$

My Canadian lecture ended with these conclusions:

- No conventional explanation for the signature e-p events has been found.

- The hypothesis that the signature e-p events come from the production of

a pair of new particles - each of mass about $2 \mathrm{GeV}$ - fits almost all the data. Only the $\theta_{\text {coll }}$ distribution is somewhat puzzling.

- The assumption that we are also detecting e-e and $\mu-\mu$ events coming from these new particles is still being tested.

I still was not able to specify the source of the e- $\mu$ events: leptons, mesons, or bosons. But I remember feeling strongly that the source was heavy leptons It would take two more years to prove that.

Table 3. Distribution of 513, 4.8-GeV, 2-Prong Events that Meet the Criteria: $p_{e}>0.65 \mathrm{GeV} / \mathrm{c}$, $\mathrm{p}_{\mathrm{u}}>0.65 \mathrm{GeV} / \mathrm{c}, \phi_{\mathrm{manl}}>20^{\circ}$

\begin{tabular}{|l|c|c|c|c|c|c|}
\hline \multicolumn{4}{|c|}{ Total Charge $=0$} & \multicolumn{3}{c|}{ Total Charge $= \pm 2$} \\
\hline Number of photons & 0 & 1 & $>1$ & 0 & 1 & $>1$ \\
\hline e-e & 40 & 111 & 55 & 0 & 0 & 0 \\
\hline e- $\mu$ & 24 & 8 & 8 & 0 & 0 & 3 \\
\hline$\mu-\mu$ & 16 & 15 & 6 & 0 & 0 & 0 \\
\hline e-h & 18 & 23 & 32 & 2 & 3 & 3 \\
\hline$\mu-h$ & 15 & 16 & 31 & 4 & 0 & 5 \\
\hline h-h & 13 & 11 & 30 & 10 & 4 & 6 \\
\hline Sum & 126 & 184 & 162 & 16 & 8 & 17 \\
\hline
\end{tabular}


Table 4. Misidentification Probabilities for a 4.8-GeV Sample (Perl 1975a)

\begin{tabular}{|l|c|c|c|}
\hline Momentum range $(\mathrm{GeV} / \mathrm{c})$ & Ph-»e & Ph->n & Ph-»h \\
\hline $0.6-0.9$ & $.130 \pm .005$ & $.161 \pm .006$ & $.709 \pm .012$ \\
\hline $0.9-1.2$ & $.160 \pm .009$ & $.213 \pm .011$ & $.627 \pm .020$ \\
\hline $1.2-1.6$ & $.206 \pm .016$ & $.216 \pm .017$ & $.578 \pm .029$ \\
\hline $1.6-2.4$ & $.269 \pm .031$ & $.211 \pm .027$ & $.520 \pm .043$ \\
\hline Weighted average using hh, ph, and ep events & $.183 \pm .007$ & $.198 \pm .007$ & $.619 \pm .012$ \\
\hline
\end{tabular}

\subsection{Steps Toward a Discovery}

\subsubsection{No Conventional Explanation}

As 1974 passed, we acquired $\mathrm{e}^{+} \mathrm{e}$ annihilation data at more and more energies, and at each of these energies there was an anomalous e-p event signal, as seen in Figure 7. Thus, I and my colleagues in the SLAC-LBL experiment became more and more convinced of the reality of the e-p events and the absence of a conventional explanation. An important factor in this growing conviction was the addition of a special muon detection system to the detector (Figure 8a), called the mиоn tower. This addition was conceived and built by Gary Feldman. Although we did not use events such as those in Figure $8 \mathrm{~b}$ in our first publication, seeing a few events like this was enormously comforting.

Finally, in December 1975, the SLAC-LBL experimenters published "Evidence for Anomalous Lepton Production in $\mathrm{e}^{+}-\mathrm{e}$ Annihilation." The final paragraph reads as follows:

We conclude that the signature e-p events cannot be explained either by the production and decay of any presently known particles or as coming from any of the well-understood interactions which can conventionally lead to an e and a $\mathrm{p}$ in the final state. A possible explanation for these events is the production and decay of a pair of new particles, each having a mass in the range of 1.6 to 2.0 $\mathrm{GeV} / \mathrm{c}^{2}$ (Perl et al. 1975b).

We were not yet prepared to claim that we had found a new charged lepton, but we were prepared to claim that we had found something new. To accentuate our uncertainty I denoted the new particle by U for "unknown" in some of our 1975-1977 papers. The name $\mathrm{T}$ was suggested to me by Petros Rapidis, who was then a graduate student and worked with me in the early 1970s on the e-p 


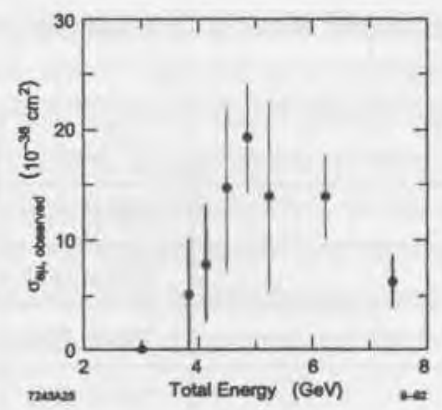

Figure 7. The observed cross section for the signature e-p events from the SLAC-LBL experiment at SPEAR. This observed cross section is not corrected for acceptance. There are 86 events with a calculated background of 22 events. (Perl et al. 1975b)

(a)
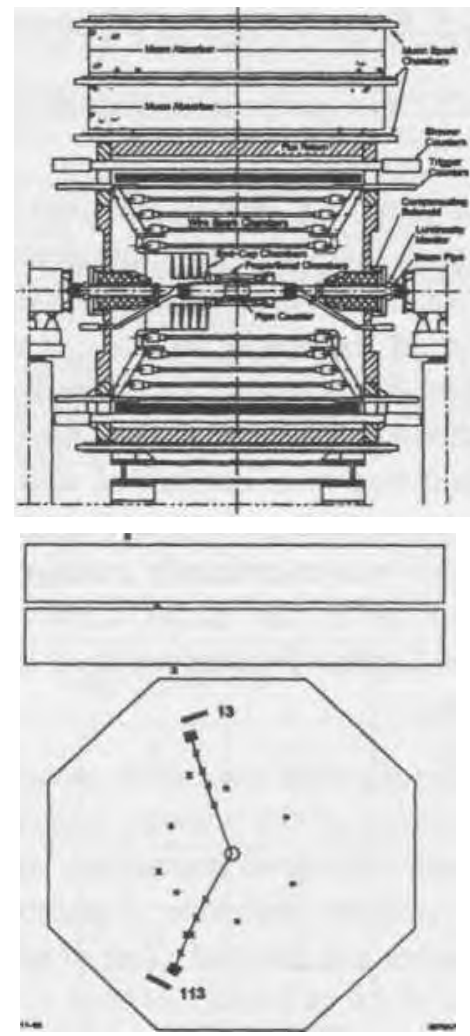

Figure 8a. The SLAC-LBL detector with the muon tower. 8b. One of the first e-p events using the tower. The $\mathrm{p}$ moves upward through the muon detector tower and the e moves downward The numbers 13 and 113 give the relative amounts of electromagnetic shower energy deposited by the $\mathrm{p}$ and $\mathrm{e}$. The six square dots show the positions of longitudinal support posts of the magnetostrictive spark chamber used for tracking. 
problem (Perl and Rapidis 1974). The letter $\mathrm{T}$ is from the Greek triton for third - the third charged lepton. Thus in 1975, twelve years after we began our lepton physics studies at SLAC, these studies finally bore fruit. But we still had to convince the world that the e-p events were significant, and we had to convince ourselves that the e-p events came from the decay of a pair of heavy leptons.

\section{If you are looking for something new in the physical sciences, try to have a reasonable way to make it and a reasonable way to find it.}

This is a good place to reflect on the elements of the research that led to the discovery of the tau. First, I had chosen a research area in which there were few investigators. Second, we had cast a wide net in studying the electron-muon problem: an attempt to photoproduce new leptons, experimental comparisons of muon-proton inelastic scattering with electron-proton inelastic scattering, and the use of the general reaction $\mathrm{e}^{+}$ $+\mathrm{e}^{-} \longrightarrow \mathrm{L}^{+}+\mathrm{L}^{-}$to try to produce a heavy lepton. Third, a new technology, the electronpositron collider, was available to carry out the $\mathrm{L}^{+} \mathrm{L}^{-}$production. Fourth, I had a good way to detect the $\mathrm{L}^{+} \mathrm{L}^{-}$production, namely the search for e-p events without photons. Fifth, I had smart, resourceful, and patient research companions. I think these are the elements that should be present in speculative experimental work: a broad general plan, specific research methods, new technology, and first-class research companions. Of course, the element of luck will in the end be dominant. I had two great pieces of luck. First, there was a heavy lepton within the energy range of the SPEAR collider. Second, the SLAC-LBL experimental apparatus was good enough to enable us to identify the e-p events and prove their existence.

\subsubsection{Is It a Lepton?}

Our first publication was followed by several years of confusion and uncertainty about the validity of our data and its interpretation. It is hard to explain this confusion a decade later when we know that $\mathrm{T}$ pair production is $20 \%$ of the $\mathrm{e}^{+} \mathrm{e}$ annihilation cross section below the $Z^{\circ}$, when тT pair events stand out so clearly at the $Z^{\circ}$.

There were several reasons for the uncertainties of that period. It was hard to believe that both a new quark (charm) and a new lepton (tau) would be found in the same narrow range of energies. Also, while the existence of a fourth quark was required by theory, there was no such requirement for a third charged lepton, so there were claims that the other predicted decay modes of tau pairs, such as e-hadron and p-hadron events, could not be found. Indeed, finding such events was just at the limit of the particle identification capability of the detectors of the mid-1970s. 
Perhaps the greatest impediment to the acceptance of the $\tau$ as the third charged lepton was that there was no other evidence for a third particle generation. Two sets of particles-u, d, e, $\nu_{\mathrm{e}}$ and $\mathrm{c}, \mathrm{s}, \mu^{-}, \nu_{\mathrm{m}}-$ seemed acceptable, a kind of doubling of particles. But why three sets? To this day, this question has no answer. It was a difficult time. Rumors kept arriving of definitive evidence against the $\tau$ : $\mathrm{e}-\mu$ events not seen, the $\tau \rightarrow \pi v$ decay not seen, theoretical problems with momentum spectra or angular distribution. With colleagues such as Gary Feldman, I went over our data again and again. Had we gone wrong somewhere in our analysis of the data? Clearly, other tau pair decay modes had to be found.

Assuming the $\tau$ to be a charged lepton with conventional weak interactions, simple and very general theory predicted the branching fractions

$$
\begin{aligned}
& \mathrm{B}\left(\tau \rightarrow v_{\mathrm{t}}+\mathrm{e}^{-}+\bar{v}_{s}\right) \approx 20 \% \\
& \mathrm{~B}\left(\tau \rightarrow v_{\mathrm{t}}+\mathrm{m}^{-}+\bar{v}_{\mu}\right) \approx 20 \% \\
& \mathrm{~B}\left(\tau \rightarrow v_{\tau}+\text { hadrons }\right) \approx 60 \% .
\end{aligned}
$$

Experimenters therefore should be able to find the decay sequences

and

$$
\begin{aligned}
& \mathrm{e}^{+}+\mathrm{e}^{-} \rightarrow \tau^{+}+\tau \\
& \tau^{+} \rightarrow \bar{v}_{\tau^{+}}+\mu^{+}+v_{\mu} \\
& \tau \rightarrow v_{\tau}+\text { hadrons, } \\
& \mathrm{e}^{+}+\mathrm{e}^{-} \rightarrow \tau^{+}+\tau \\
& \tau^{+} \rightarrow \bar{v}_{\tau}+\mathrm{e}^{+}+v_{\mathrm{e}} \\
& \tau \rightarrow v_{\tau}+\text { hadrons. }
\end{aligned}
$$

The first sequence, Eqs. 13, would lead to anomalous muon events

$$
\mathrm{e}^{+}+\mathrm{e}^{-} \rightarrow \mu^{ \pm}+\text {hadrons }+ \text { missing energy, }
$$

and the second, Eqs. 14, would lead to anomalous electron events

$$
\mathrm{e}^{+}+\mathrm{e}^{-} \rightarrow \mathrm{e}^{ \pm}+\text {hadrons }+ \text { missing energy. }
$$

\subsubsection{Anomalous Muon Events and a Sequential Heavy Lepton}

The first advance beyond the e- $\mu$ events came with three different demonstrations of the existence of anomalous $\mu$-hadron events:

$$
\mathrm{e}^{+}+\mathrm{e}^{-} \rightarrow \mu^{ \pm}+\text {hadrons }+ \text { missing energy. }
$$


The first and very welcome outside confirmation of anomalous muon events came in 1976 from another SPEAR experiment by Cavilli-Sforza et al. (1976). The paper was titled "Anomalous Production of High-Energy Muons in $\mathrm{e}^{+}+\mathrm{e}^{-}$Collisions at $4.8 \mathrm{GeV}$." I have in my files a June 3, 1976, SLAC-LBL note by Gary Feldman discussing g events using the muon identification tower of the SLAC-LBL detector (see Figure 8a). For data acquired above $5.8 \mathrm{GeV}$, he found the following:

Correcting for particle misidentification, this data sample contains $8 \mathrm{e}-\mathrm{g}$ events and $17 \mathrm{~g}$-hadron events. Thus, if the acceptance for hadrons is about the same as the acceptance for electrons, and these two anomalous signals come from the same source, then with large errors, the branching ratio into one observed charged hadron is about twice the branching ratio into an electron. This is almost exactly what one would expect for the decay of a heavy lepton.

This conclusion was published in the paper "Inclusive Anomalous Muon Production in ${ }^{\text {e+e- }}$ Annihilation," by Feldman et al. (1977). The most welcome confirmation, because it came from an experiment at the DORIS $\mathrm{e}^{+} \mathrm{e}^{-}$storage ring, was from the PLUTO experiment. In 1977, the PLUTO collaboration published "Anomalous Muon Production in $\mathrm{e}^{+} \mathrm{e}^{-}$ Annihilation as Evidence for Heavy Leptons," (Burmester et al. 1977); Figure 9 is from that paper. PLUTO was also a large-solid-angle detector, and so, for the first time, we could fully discuss the art and technology of $\mathrm{T}$ research with an independent set of experimenters, our friends Hinrich Meyer and Eric Lohrman of the PLUTO collaboration.

With the finding of $\mu$-hadron events, I was convinced I was right about the existence of the $\tau$ as a sequential heavy lepton. Yet there was much to disentangle: it was still difficult to demonstrate the existence of anomalous e-hadron events, and the major hadronic decay modes

$$
\begin{aligned}
& \tau \rightarrow v_{\tau}+\pi \\
& \tau \rightarrow v_{\tau}+\rho^{-}
\end{aligned}
$$

had to be found.

The demonstration of the existence of anomalous electron events,

$$
\mathrm{e}^{+}+\mathrm{e}^{-}-\mathrm{e}^{ \pm}+\text {hadrons }+ \text { missing energy, }
$$

required improved electron identification in the detectors. A substantial step forward was made by the new DELCO detector at SPEAR (Kirkby 1977; Bacino et al. 1978). In his talk at the 1977 Hamburg Photon-Lepton Conference, Kirkby 
stated, "A comparison of the events having only two visible prongs (of which only one is an electron) with the heavy lepton hypothesis shows no disagreement. Alternative hypotheses have not yet been investigated."

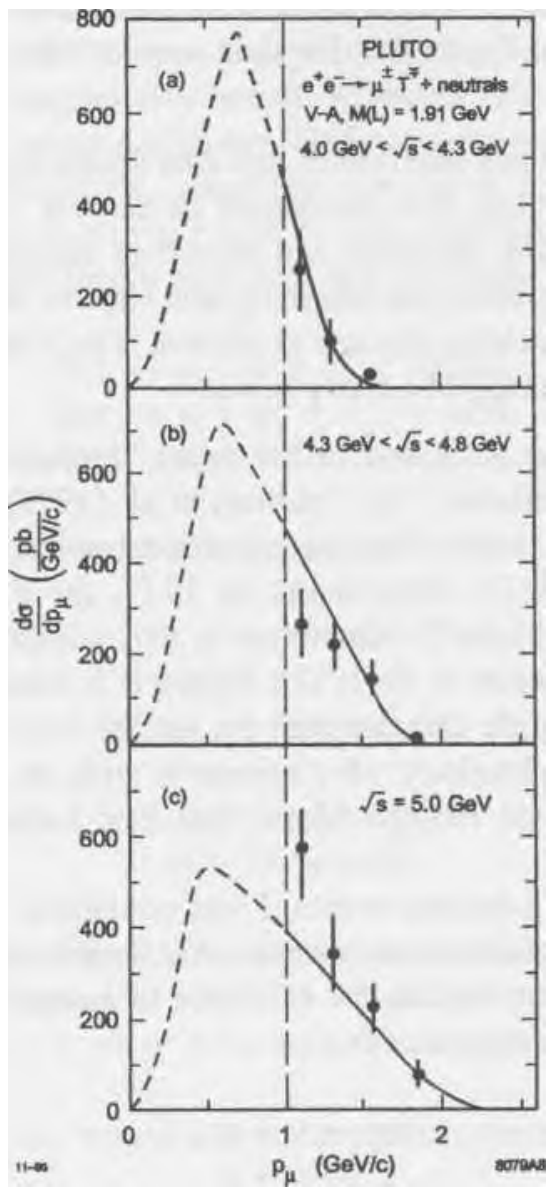

Figure 9. The momentum spectra of p's from anomalous muon events found by the PLUTO experimenters using the DORIS e'e storage ring (Burmester et al. 1977).

The SLAC-LBL detector was also improved by Group E from SLAC and a Lawrence Berkeley Laboratory group led by Angela Barbaro-Galtieri; some of the original SLACLBL experimenters had gone off to begin to build the Mark II detector. We installed a wall of lead-glass electromagnetic shower detectors in the SLAC-LBL detector (see Figure 10). This led to the important paper by Barbaro-Galtieri et al. (1977b). The abstract read as follows: 
We observe anomalous $\mathrm{e}-\mu$ and $\mathrm{e}$-hadron events in $\mathrm{e}^{+}+\mathrm{e}^{-} \rightarrow \tau^{+}+\tau$ with subsequent decays of $\tau^{ \pm}$into leptons and hadrons. Under the assumption that they come only from this source, we measure the branching ratios $\mathrm{B}\left(\tau \rightarrow \mathrm{e} \mathrm{v}_{\mathrm{e}} \mathrm{v}_{\tau}\right)=(22.4 \pm 5.5) \%$ and $\mathrm{B}(\tau \rightarrow \mathrm{h}+$ neutrals $)=(45 \pm 19) \%$.

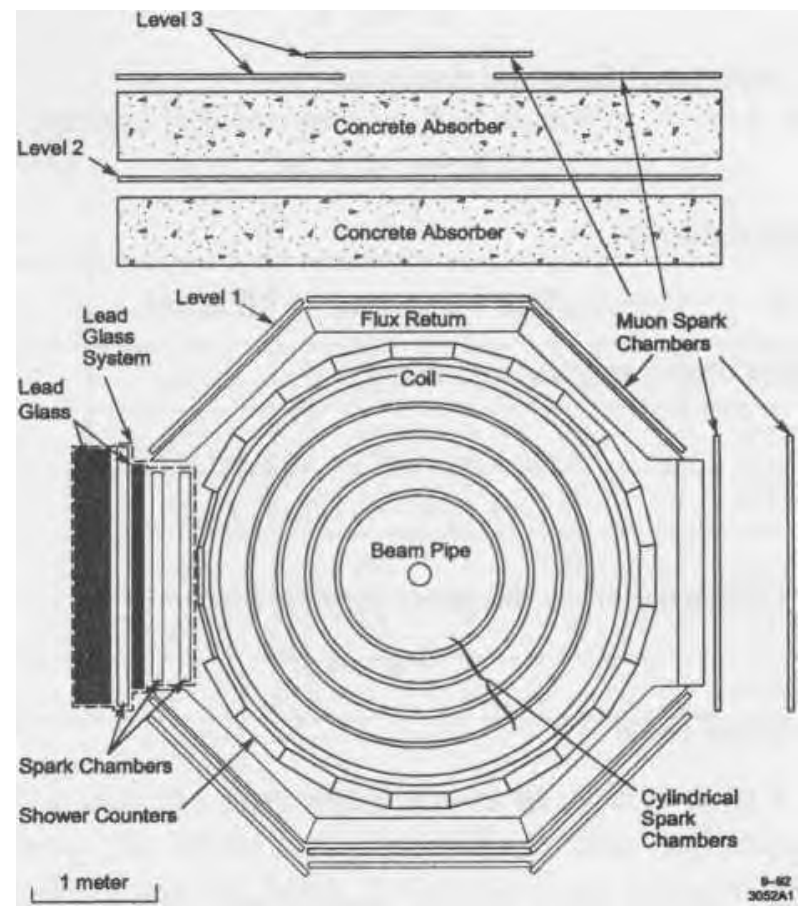

Figure 10. The "lead-glass wall" modification of the SLAC-LBL detector used at SPEAR to find anomalous electron events.

By the time of the 1977 Photon Lepton Conference at Hamburg, I was able to report (Perl 1977) the following:

- All data on anomalous $\mathrm{e}-\mu, \mathrm{e}-\mathrm{x}$, e-e, and $\mu-\mu$ events produced in $\mathrm{e}^{+} \mathrm{e}^{-}$ annihilation are consistent with the existence of a mass $1.9 \pm 0.1 \mathrm{GeV} / \mathrm{c}^{2}$ charged lepton, the $\tau$.

- These data cannot be explained as coming from charmed particle decays.

- Many of the expected decay modes of the $\tau$ have been seen. A very important problem is the existence of the $\tau \rightarrow v_{\tau} \pi$ decay mode.

- Semi-leptonic decay modes and the search for $\tau \rightarrow \nu_{\tau} \pi$ and $\tau \rightarrow \nu_{\tau} \rho^{-}$.

The anomalous muon and anomalous electron events had shown that the total decay rate of the $\mathrm{T}$ into hadrons, that is, the total semi-leptonic decay rate, 
was about the right size. But if the $\tau$ was indeed a sequential heavy lepton, two substantial semi-leptonic decay modes had to exist: $\tau \rightarrow \nu_{\tau} \pi$ and $\tau \rightarrow v_{\tau} \rho^{-}$.

\section{First, the branching fraction for}

$$
\tau \rightarrow v_{\tau} \pi
$$

could be calculated from the decay rate for

$$
\pi \rightarrow \mu^{-}+\bar{v}_{\mu}
$$

and was found to be

$$
\mathrm{B}\left(\tau \rightarrow v_{\tau} \pi^{-}\right) \approx 10 \% .
$$

Second, the branching fraction for

$$
\begin{aligned}
\tau & \rightarrow v_{\tau}+\rho^{-} \rightarrow v_{\tau}+\pi^{-}+\pi^{\circ} \\
& \rightarrow v_{\tau}+\pi^{-}+\gamma^{+}+\gamma
\end{aligned}
$$

could be calculated from the cross section for

$$
\mathrm{e}^{+}+\mathrm{e}^{-} \rightarrow \rho^{\circ}
$$

and was found to be

$$
\mathrm{B}\left(\tau \rightarrow v_{\tau} \rho^{-}\right) \approx 20 \%
$$

One of the problems in the years 1977-1979 in finding the modes in Eqs. 19a and 20a was the poor efficiency for photon detection of the early detectors. If the $\gamma$ 's in Eq. $20 \mathrm{a}$ are not detected, then the $\pi$ and $\rho$ modes are confused with each other. Probably the first separation of these modes was achieved using th $<$ LeadGlass Wall detector. As reported at the Hamburg Conference by Angelin. Barbaro-Galtieri (1977a),

$$
\mathrm{B}\left(\tau \rightarrow \nu_{\tau} \pi^{-}\right) / \mathrm{B}\left(\tau^{-} \rightarrow \nu_{\tau} \rho^{-}\right)=0.44 \pm 0.37 .
$$

Gradually, the experimenters understood the photon detection efficiency of their experiments. In addition, new detectors with improved photon detection efficiency (such as the Mark II) were put into operation. In our collaboration,

the first demonstration that $\mathrm{B}\left(\tau \rightarrow \nu_{\tau} \pi\right)$ was substantial came from Gail Hanson in an internal note dated March 7, 1978.

Within about a year, the $\tau \rightarrow v_{\tau} \pi^{-}$decay mode had been detected and measured by experimenters using the PLUTO detector, the DELCO detector, 
the Lead-Glass Wall detector, and the new Mark II detector. These measurements were summarized (Table 5) by Gary Feldman (1978) in a review of $\mathrm{e}^{+}+\mathrm{e}^{-}$annihilation physics at the XIX International Conference on High-Energy Physics. Although the average of the results in Table 5 is two standard deviations

smaller than the present value of $(11.1 \pm 0.2) \%$, the $\tau \rightarrow v_{\tau} \pi$ mode had been found.

Table 5. The various measured branching fractions B in percent for $\tau \rightarrow \pi v_{\tau}$ in late 1978 (Feldman 1979).

\begin{tabular}{|l|c|c|c|c|}
\hline \multicolumn{1}{|c|}{ Experiment } & Mode & Events & Background & $\mathrm{B}(\tau \rightarrow \pi v)$ \\
\hline SLAC-LBL & $\mathrm{x} \pi$ & $\approx 200$ & $\approx 70$ & $9.3 \pm 1.0 \pm 3.8$ \\
\hline PLUTO & $\mathrm{x} \pi$ & 32 & 9 & $9.0 \pm 2.9 \pm 2.5$ \\
\hline DELCO & $\mathrm{e} \pi$ & 18 & 7 & $8.0 \pm 3.2 \pm 1.3$ \\
\hline \multirow{2}{*}{ Mark II } & $\mathrm{x} \pi$ & 142 & 46 & $8.0 \pm 1.1 \pm 1.5$ \\
\cline { 2 - 5 } & $\mathrm{e} \pi$ & 27 & 10 & $8.2 \pm 2.0 \pm 1.5$ \\
\hline Average & & & & $8.3 \pm 1.4$ \\
\hline
\end{tabular}

The year 1979 saw the first publications of $\mathrm{B}\left(\tau \rightarrow v_{\tau} \rho^{-}\right)$. The DASP Collaboration, using the DORIS $\mathrm{e}^{+}+\mathrm{e}^{-}$storage ring, reported $(24 \pm 9) \%$ (Brandelik et al. 1979), and the Mark II Collaboration reported $(20.5 \pm 4.1) \%$ (Abrams et al, 1979). The measurements are crude, but they agree with the $20 \%$ estimate in Eq. 20c. The present value is $(24.8 \pm 0.2) \%$.

By the end of 1979 , all confirmed measurements agreed with the hypothesis that the $\tau$ was a lepton produced by a known electromagnetic interaction and that, at least in its main modes, it decayed through the conventional weak interaction. And so ends the sixteen-year history, 1963 to 1979 , of the discovery of the tau lepton and the verification of that discovery.

\section{From the Tau to Fractional Electric Charge and Very Small Drops, 1980s and 1990s}

To my astonishment, no more heavy leptons have been seen. The best search was conducted in Europe, where they searched up to $95 \mathrm{GeV}$, a big jump in range from the tau, which has a mass of $1.8 \mathrm{GeV}$. I keep using this table because it represents what we knew by 1980 and in 1999 . 


\begin{tabular}{|c|l|c|l|}
\hline \multicolumn{4}{|c|}{ Leptons in 1996} \\
\hline \multirow{2}{*}{ Generation } & \multicolumn{1}{|c|}{ Particle } & Electric Charge & \multicolumn{1}{|c|}{ Mass } \\
\hline 1 & $\begin{array}{l}\text { Electron }(e) \\
\text { Electron neutrino }\left(v_{e}\right)\end{array}$ & -1 & About $0.5 \mathrm{MeV}$ \\
\cline { 3 - 4 } & Muon $(\mu)$ & -1 & Close to 0 or 0 \\
\hline \multirow{2}{*}{2} & Muon Neutrino $\left(v_{\mu}\right)$ & 0 & About $105 \mathrm{MeV}$ \\
\cline { 3 - 4 } & Tau $(\tau)$ & -1 & Close to 0 or 0 \\
\hline \multirow{2}{*}{3} & Tau neutrino $\left(v_{\tau}\right)$ & 0 & About $1780 \mathrm{MeV}$ \\
\cline { 3 - 4 } & & & Close to 0 or 0 \\
\hline
\end{tabular}

The problems I started working on in the 1960s have not been solved. We have the electron, the muon, and the tau. We do not understand why there are three, not more-why their masses are so different, or even what sets mass. I'd love to do more on the tau, on the heavy leptons, but I simply don't know where to go, and so I have moved on.

About six years ago, I became interested in looking for fractionally charged particles. It has been known for about 80 years that all the elementary particles that can be separated have integer, not fractional, charges in units of the electron charge. The only particles with fractional charges are quarks but they have never been isolated because they are bound into protons, neutrons, and pions. Klaus Lackner and Gordon Shaw pointed out that with new technology we could make a much improved search for fractional charge particles (Mar et al. 1966).

Our ongoing research interest is in searching in bulk matter for fractional charge elementary particles produced in the early universe - particles whose properties preclude discovery using accelerators or other traditional search methods of high-energy physics. When a star forms, these particles will be pulled into the star as either free particles or attached to light nuclei. Upon the disintegration of the star, these particles, either free or attached to nuclei, would be expelled into space. Our hope is that these particles would eventually be swept up in the formation of the solar system.

It is best to look at unrefined materials such as unprocessed terrestrial minerals and meteorites from asteroids, because it is quite possible that in refined materials, such as iron or niobium, the atom with fractional charge has been lost in the refining process (Lackner and Zweig 1985).

There are two benefits in searching for fractional charge particles through this path. First, the early universe production processes for such particles are very general, especially with respect to the mass range. Second, even an upper 
limit on the occurrence of fractional charge particles tells us about the constituents of the solar system in particular and the universe in general.

The present form of the experiment is a very simple idea (see Figure 11 below). The whole apparatus is quite small, requiring two electric plates, a drop generator, and an optical system; the drop generator and optical system are not shown in the figure. We make a drop and let it fall in air. The drops are small, ranging from 7 to 50 microns. With Stokes' law, for particles this size, they quickly reach terminal velocity. The terminal velocity, $\mathrm{v}$, is proportional to the force; thus the dynamics are that of Aristotle, not Newton.

If a drop is charged, the electric field moves the drop horizontally with terminal velocity component $v_{c}$ Meanwhile, gravity moves the drop downward with vertical terminal velocity $\mathrm{v}_{\mathrm{g}}$. We look at tens of millions of drops and have a beautiful optical system with a CCD camera for doing this. The charge measurement works as follows. Using a computer to analyze the output of the CCD camera, we measure the drop trajectory as a function of time. We also measure the drop radius using the drop image from the CCD camera. Since we know the horizontal component of the velocity, the drop radius, and the viscosity of air, we can calculate the drop charge. Of course, this is all done automatically by the computer. We measure the charge of a drop to about a $40^{\text {th }}$ of an electron charge.

We use drops of various sizes. We've worked on pure liquids, mostly silicon oil, and we have not found any fractionally charged particles (Perl and Lee 1997). But pure liquids, especially oils, are refined, so now we're working in drops containing suspended groundup minerals or meteorites. We have been working on how to do this and basically have it working after learning a great deal from the industrial chemistry of suspensions and surfactants.

We're the only ones left working in this field. It is completely speculative. It's great fun to work in a very small group like this. That's the good part. The bad part is that we have to make every mistake in the field ourselves. If there were three or four other groups, we could call other people and say "We're saving problems grinding things fine enough." The best situation is where there are three to four groups and you're in friendly competition. So we're a little isolated.

To our great surprise, out of this speculative, pure science experiment have come all sorts of possible applications. For example, we make these drops just the way that ink jet and bubble jet printers work. Because we had to deal with high-viscosity oils and suspensions, we had to develop our own droppers with micromachining. We stumbled into quite a nice technology. We have received DOE technology maturation funding and have some patents through Stanford University. We have gone into a number of areas beyond our knowledge and strengths and have more applications than we can handle. 
One fascinating application is for gene chips. With gene chips, you put down a thousand different samples of DNA and then test which one the DNA belongs to. The best technology for doing this is almost like quill pens, and ours may not be competitive. Our idea is to develop droppers that will eject DNA because we can make smaller drops this way. Others have tried and failed but it's hard to find out about this application because it's proprietary in the gene chip business.

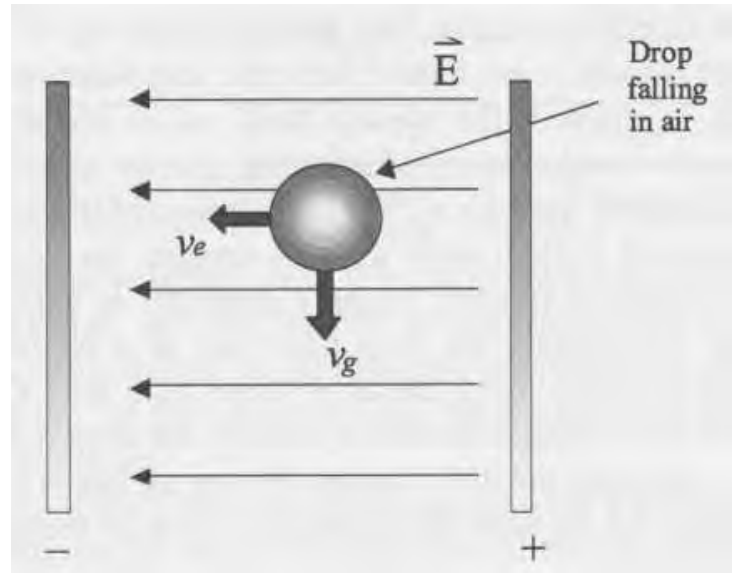

From Stokes' law

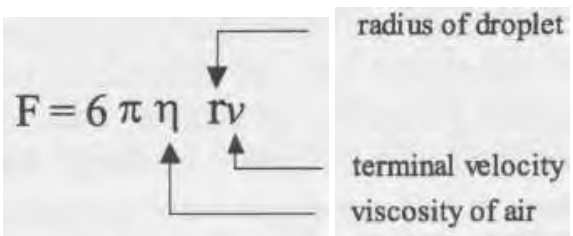

Obtain charge on droplet from

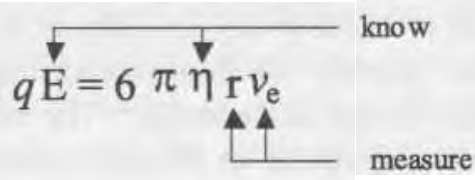

Also

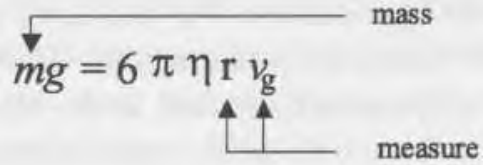

Figure 11. Measuring the drop. 
Another application is for ink jet printers. The secret of making money in printers is in the ink. At Hewlett Packard, Epson, and Canon, the ink is tuned very carefully to the printer and papers.

We have stumbled into other application areas. For example, we can measure the evaporation of drops. If you make a whole sequence of drops, they will influence each other hydrodynamically and this is of interest in combustion engineering. We have a dropper that can make any pattern desired. Mixing up a lot of things in thousands of samples is of interest in combinatorial chemistry.

One of the things we've discovered in passing on a technology is that it has to be a one-way street. For example, a company is always afraid that its proprietary information will be spread around and used by its competitors. We are an open laboratory and we will keep secrets, but not purposefully. The problem is that we often don't know what the user really wants, and the user can't tell us because of proprietary interests.

\section{Observations on Experimenters, Experiments, and Theory}

In this paper, I have tried to pass on what I have learned in fifty years of science and engineering:

Follow your own personality in science and engineering.

Be prepared to do work that is boring or that requires skills in which you are not particularly gifted.

Do not try to predict the future of a technology used in engineering or science.

Be aware of the emotional roller coaster of research.

Use your own ideas for and in experiments.

You don't have to be a fast talker or thinker; in fact, it is best to avoid such people.

Choose the group or department where you will have the most freedom.

You don't have to know everything-you can learn a technology or subject when you need it.

Stay away from lines of research where very smart and competent people are working.

Understand the art of obsession in science-focus on the experiment but know when to give it up.

For every good idea expect five or ten bad ideas.

If you are looking for something new in the physical sciences, try to have a reasonable way to make it and a reasonable way to find it. 


\section{$1 A$ Final remarks}

\section{Choose companions in research who are smarter than you.}

Choose companions who are smarter than you, who think faster than you but say little, and who don't cut your ideas down until it becomes obvious to you that they won't work.

\section{You should not know more than you have to.}

How much should you know? Not more than you have to. You have to keep changing and learning.

\section{Do not confuse research with administrative or committee work.}

I would also caution you not to confuse research with administrative or committee work. These are not the same thing. There is no way around it - the world is a complicated place and administrative work, such as for safety, has to be done. You need committees in science but nature knows what's going on and the committee may not. Suppose you're having a problem with too much noise in a big detector and you convene a committee of two to three experts; the committee may come to a decision and make recommendations on what should be investigated. One expert may say that the ground loops were done wrong, another that integrated circuits should not have been used, and another that it's electromagnetic interference from another person's equipment. But these may have nothing to do with what is causing the noise. The problem may actually be that there is a water leak causing electromagnetic problems and the noise.

\section{Intellectual hierarchies in science are a pernicious doctrine.}

I would add that intellectual hierarchies in science are a pernicious doctrine. At the top are the theoretical physicists (and it's best if they are working in string theory, pure mathematics), next are the experimentalists working in pure science, followed by those working in applied science, and then come the device makers, the engineers, and so on. In my work, I never draw a distinction between these as long as the problem is interesting.

\section{Follow experimental science where it goes and do not be driven by the crowd.}

It is important not to be driven by what the crowd is doing. Working in your own direction is always good. Over ninety-nine percent of the time the crowd is right but, sometimes, they're wrong and you're working in the other area and that's great. One has to balance these things. 
My final remark to the young women and men going into experimental science is to pay little attention to today's speculative physics ideas of my generation. After all, if my generation had any really good speculative ideas, we would be carrying these ideas out ourselves.

\section{References}

Abrams, G. S., et al., 1979, Phys. Rev. Lett. 43, 1555.

Alles-Borelli, V., et al., 1970, Lett. Nuovo Cimento I-V, 1156.

Bacino, W., et al., 1978, Phys. Rev. Lett. 41, 13.

Barbaro-Galtieri, A., 1977a, "Proceedings of the 1977 International Symposium on Lepton and Photon Interactions at High Energies," edited by F. Gutbrod (Hamburg 1977), p. 21.

Barbaro-Galtieri, A., et al., 1977b, Phys. Rev. Lett. 39, 1058.

Barber, W. C., et al., 1966, Phys. Rev. Lett. 16, 1127.

Bama, A., et al., 1968, Phys. Rev. 173, 1391.

Bemardini, M., 1973, Nuovo Cimento 17A, 383.

Bjorken, J. D., and C. H, Llewellyn-Smith, 1973, Phys. Rev. Lett. D7, 887.

Brandelik, R., et al., 1979, Z. Phys. Cl., 233.

Braunstein, T., et al., 1972, Phys. Rev. D6, 106.

Burmester, J., et al., 1977, Phys. Lett. 68B, 297.

Cabibbo, N, and R. Gatto, 1961, Phys. Rev. 124, 1577.

Camilleri, L., et al., 1969, Phys. Lett. 23, 153.

Cavalli-Sforza, M., et al., 1976, Phys. Rev. Lett. 36, 558.

Charpak, G., et al., 1962, Phys. Lett. 1, 16.

Damouth, D. E., L. W. Jones, and M. L. Perl, 1963, Phys. Rev. Lett. 11, 287.

Danby, G., et al., 1962, Phys. Rev. Lett. 9, 36.

Ellsworth, R. W., et al., 1960, Phys. Rev. 165, 1449.

Feldman, G. J., et al., 1977, Phys. Rev. Lett. 38, 117.

Feldman, G. J., 1978, "Proceedings of the XIX International Conference on High-Energy

Physics," edited by S. Hounma, M. Kawaguchi, and H. Miyazawa (Tokyo, 1978).

Feldman, G. J., et al., 1982, Phys. Rev. Lett. 48, 66.

Hanson, G., 1978, SLAC-LBL Collaboration internal note, March 7, 1978.

Howard, F. T., 1967, "Proceedings of the Sixth International Conference on High Energy

Accelerators," edited by R. A. Mack (Cambridge, 1967), p. B43.

Kirkby, J., 1977, "Proceedings of the 1977 International Symposium on Lepton and Photon

Interactions at High Energies," edited by F. Gutbrod (Hamburg, 1977), p. 3. Kirkby, J.,

and J. A. Rubio, 1992, Particle World 3, 77.

Kostoulas, I., et al., 1974, Phys. Rev. Lett. 32, 489.

Kreisler, M. N., et al., 1966, Phys. Rev. Lett. 16, 1217.

Lackner, K. S., and G. Zweig, 1982, "The Search for Fractionally Charged Particles," published in the Proceedings of the Fifth International Conference on Particle Physics at Vanderbilt, May 1982, Los National Laboratory report LA-UR 82-2988.

Lai, K. W., L. W. Jones, and M. L. Perl, 1961, "Proceedings of the 1960 International Conference on Instrumentation in High-Energy Physics," edited by C. E. Mauk, A. H. Rosenfeld, and R. K. Wakerling (Interscience, New York, 1961), p. 186. 
Larsen, R. M., et al., 1971, SLAC Proposal SP-2.

Lipmanov, E. M,, 1964, JETP Lett. 19, 1291.

Low, F. E,, 1965, Phys. Rev. Lett. 14, 238.

Mar, N. M., et al., 1996, "Improved search for elementary particles with fractional electric charge," Phys. Rev D, Part. Fields, 53, no.ll, 6017.

Montanet, L., et al., 1994, "Review of Particle Properties," in Phys. Rev D50, 1173. Okun, L. B., 1965, Soviet Phys. JETP Lett. 20, 1197.

O’Neil, G. K., et al., 1958, HEPL Report RX-1486.

Orito, S., et al., 1974, Phys. Lett. 48B, 165.

Perl, M. L., I. I. Rabi, and B. Senitzky, 1955, Phys. Rev 97, 838.

Perl, M. L., L. W. Jones, and C. C. Ting, 1963, Phys. Rev 132, 1252.

Perl, M. L., 1971, Phys. Today, July, p. 34.

Perl, M. L., 1975a, "Proceedings of the Canadian Institute of Particle Physics, Summer School," edited by R. Heinzi and B. Margolis (McGill University, Montreal, 1975).

Perl, M. L., et al., 1975b, Phys. Rev Lett. 35, 1489.

Perl, M. L., 1977, "Proceedings of the 1977 International Symposium on Lepton and Photon Interactions at High Energies," edited by R. Gutbrod (Hamburg, 1977), p. 145.

Perl, M. L., and P. Rapidis, 1974, SLAC-PUB-1496, unpublished.

Perl, M. L., and L. V. Beers, 1994, "Proceedings of the Workshop on Tau-Charm Factory in the Era of B-Factories and CESR," SLAC-451, edited by L. V. Beers and M. L. Perl (SLAC, Stanford, 1994).

Perl, M. L., 1996, "Reflections on Experimental Science,” (World Scientific, Singapore, 1996).

Perl, M. L., 1996, "Reflections on the Discovery of the Tau Lepton," Les Prix Nobel reprint, Norstedts Tryckeri, Stockholm, Sweden.

Perl, M. L., and E. R. Lee, 1997, "The search for elementary particles with fractional electric charge and the philosophy of speculative experiments," Am. J. Phys. 65, no.8, 698-706.

Reines, F., and C. L. Cowan, Jr., 1953, Phys. Rev 92, 830.

Ritson, D., S. Berman, A. Boyarski, F. Bulos, E. L. Garwin, W. Kirk, B. Richter, and M. Sands, 1964, "Proposal for a High-Energy Electron-Positron Colliding-Beam Storage Ring at the Stanford Linear Accelerator Center" (SLAC, Stanford, 1964).

Rothe, K. W, and A. M. Wolsky, 1969, Nucl. Phys. B10, 241.

Thacker, H. B., and J. J. Sakurai, 1971 Phys. Lett. 36B, 103.

Toner, W. T., et al., 1972, Phys. Lett. 36B, 251.

Tsai, Y.-S., 1971, Phys. Rev. D4, 2821.

Tsai, Y.-S., and A. C. Hearn, 1965, Phys. Rev 140B, 721.

Tsai, Y.-S., and V. Whitis, 1966, Phys. Rev. 149, 1348.

Voss, G., 1994, "Proceedings of the International Conference on the History of Original Ideas and Basic Discoveries in Particle Physics," edited by H. Newman (Sicily, 1994).

Weinstein, A. J., and R. Stroynowski, 1993, Ann. Rev. Nucl. Part. Sci. 43, 457. Wilkinson,

D., and H. R Crane, 1963, Phys. Rev. 130, 852.

Zel'dovich, Ya B., 1963, Sov. Phys. Usp. 5, 931. 